\title{
Variabilidad en la medición del índice acetabular
}

\author{
ANGÉLICA IBÁÑEZ L. ${ }^{1}$, CONSTANZA RAMÍREZ M. ${ }^{2}$, FELIPE HODGSON O. ${ }^{1}$, \\ JOAQUÍN VALENZUELA V. ${ }^{2}$, LORENA KARZULOVIC B. ${ }^{3}$, \\ JORGE LLANOS C. ${ }^{4}$, GUILLERMO IZQUIERDO P. ${ }^{5}$, MACARENA VALDÉS S. ${ }^{6}$
}

1. Traumatólogo. Departamento de Traumatología y Ortopedia, Pontificia Universidad Católica de Chile. Santiago, Chile.

2. Residente de Traumatología y Ortopedia, Pontificia Universidad Católica de Chile. Santiago, Chile.

3. Residente de Pediatría, Pontificia Universidad Católica de Chile. Santiago, Chile.

4. Radiólogo. Departamento de Radiología, Pontificia Universidad Católica de Chile. Santiago, Chile.

5. Interno de $7^{\circ}$ año de Medicina, Pontificia Universidad Católica de Chile. Santiago, Chile.

6. Tecnólogo Médico, Unidad de investigación. Departamento de Traumatología y Ortopedia, Pontificia Universidad Católica de Chile. Santiago, Chile.

\begin{abstract}
Variability in measurement of acetabular index

Developmental dysplasia of the hip (DDH) is a spectrum of diseases ranging from frank dislocation of the hip to mild acetabular dysplasia. DDH screening for detection is performed routinely in our country using pelvic $\mathrm{x}$-ray at 3 months of age. The radiographic measured acetabular index is used to evaluate the dysplastic hip, at initial presentation and during follow-up. Objective: Evaluation of the intra- and inter-observer variability, among medical professionals, when measuring acetabular index. Methods: Four reviewers (a children orthopedic surgeon, a general practitioner, a pediatrician and a radiologist) performed acetabular index measurement in 100 radiographs (200 hips), on three occasions, separated each by one month (600 total measurements). An independent observer evaluated the measurement reproducibility. The intra-class correlation coefficient to determine significant differences was used. Results: The intra-observer variability was less than the inter-observer variability. The intra-observer variability was similar among the different assessors, \pm 1.5 degrees. The inter-observer variability was \pm 3.4 degrees. Conclusions: A high concordance among measurements was reported, evidencing a high reproducibility of the acetabular index; this index is a reliable method for the diagnosis and follow-up of acetabular dysplasia.

(Key words: Developmental dysplasia of the hip (DDH), acetabular index, intra-observer variability, interobserver variability, radiography).

Rev Chil Pediatr 2013; 84 (2): 160-165
\end{abstract}

Recibido el 29 de febrero de 2012, devuelto para corregir el 26 de marzo de 2012, segunda versión el 13 de mayo de 2012 , tercera versión el 20 de agosto de 2012, aceptado para publicación el 29 de octubre de 2012.

Este trabajo no contó con financiamiento.

Premio Primer lugar Categoría Becado 52 Congreso Chileno de Pediatría Concepción 2011.

Este trabajo cumple con los requisitos sobre consentimiento /asentimiento informado, comité de ética, financiamiento, estudios animales y sobre la ausencia de conflictos de intereses según corresponda.

Correspondencia a:

Constanza Ramírez M.

E-mail:conypetrova@gmail.com 


\section{RESUMEN}

Introducción: La Displasia del desarrollo de la cadera (DDC) es un espectro de enfermedades que abarca desde la luxación franca de la cadera hasta la displasia acetabular leve. El screening de detección de DDC se realiza de rutina en nuestro país, mediante una radiografía de pelvis a los 3 meses. El índice acetabular medido en estas radiografías se utiliza para evaluar la cadera displásica, tanto en la presentación inicial como durante el seguimiento posterior. Objetivo: Evaluar la variabilidad tanto intra como inter observador en la medición del índice acetabular, entre profesionales médicos. Material y Métodos: Cuatro evaluadores (un cirujano-ortopédico infantil, un médico general, un pediatra y un radiólogo) realizaron la medición del índice acetabular en 100 radiografías de screening (200 caderas), en tres ocasiones, separadas por un mes cada una (600 mediciones totales). Un observador independiente evaluó la reproductibilidad en la medición. Se utilizó el coeficiente de correlación intraclase para determinar diferencias significativas. Resultados: La variabilidad intra observador fue menor que la interobservador. La variabilidad intra observador fue similar para los diferentes evaluadores, $\pm 1,5^{\circ}$. La variabilidad inter observador fue de $\pm 3,4^{\circ}$. Conclusiones: Demostramos una alta concordancia entre las mediciones, determinando una alta reproductibilidad del índice acetabular. El índice acetabular es un método seguro para el diagnóstico y seguimiento de displasia acetabular.

(Palabras clave: Displasia desarrollo cadera (DDC), índice acetabular, variabilidad intraobservador, variabilidad interobservador, radiografía).

Rev Chil Pediatr 2013; 84 (2): 160-165

\section{Introducción}

La displasia del desarrollo de la cadera (DDC), es una de las patologías ortopédicas más frecuentes en la población pediátrica, afectando a un 0,1 a $3 \%$ de la población ${ }^{1}$. Corresponde a un amplio espectro de anormalidades que involucran a todos los componentes mesodérmicos de esta articulación. Su severidad es variable, abarcando desde la displasia acetabular leve hasta la luxación franca de la cadera.

En Chile su incidencia se estima en 1 cada 500 a 600 recién nacidos vivos (RNV) para las formas de subluxación y luxación, lo que se traduce en 500 casos al año en el país. Dado que en Chile los RNV son alrededor de 250.000 anuales (INE 2009), si consideramos las alteraciones leves del desarrollo de la cadera, la incidencia llega al 2 a 3\% de los lactantes, es decir 5.000 a 7.500 niños $^{2}$.

El pronóstico de esta patología depende de un diagnóstico precoz y un tratamiento oportu$\mathrm{no}^{3}$, por lo que la imagenología (ecografía y/o radiología) es fundamental para el diagnóstico. La importancia del diagnóstico precoz ha llevado a la implementación en nuestro país de las garantías explícitas de salud (GES) para la confirmación y tratamiento oportuno de la
DDC, las cuales están en funcionamiento desde el año 2010.

Aunque la evidencia es controvertida respecto al valor de la ecografía sistemática ${ }^{3-6}$ entre los especialistas nacionales hay consenso en realizarla en niños que tienen factores de riesgo ${ }^{6}$. Es un examen muy apropiado para los primeros meses de vida y es superior a la radiografía ya que permite visualizar la cabeza femoral cartilaginosa y el acetábulo. La sensibilidad del examen es cercana al 90\% y su especificidad alcanza al 97\%. El valor de este examen, está dado por la posibilidad de diagnóstico precoz desde la segunda semana de vida y por ser inocuo ${ }^{4}$, sin embargo, requiere de un equipamiento que no está disponible en todos los centros de salud en nuestro medio, y es además un método operador dependiente, que requiere de un observador experimentado.

Sin embargo, el esquema de screening en nuestro país, se lleva a cabo mediante la toma de una radiografía de pelvis anteroposterior (AP) a los 3 meses de edad ${ }^{6}$, la que requiere de una correcta evaluación por parte del equipo médico.

Entre los parámetros evaluados en la radiografía de pelvis se incluyen el arco de Shenton, el índice acetabular y la línea de Perkins-Ombredanne, siendo el índice acetabular el más 


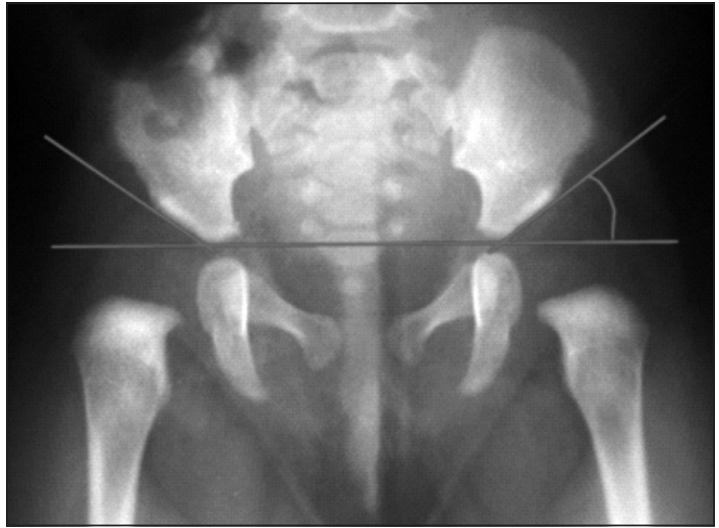

Figura 1. Medición de índice acetabular.

utilizado para la toma de decisiones terapéuti$\operatorname{cas}^{7}$. El índice acetabular (o ángulo acetabular) corresponde al ángulo formado por la línea de Hilgenreiner y una línea tangente al acetábu$10^{8}$ (figura 1). Esta medición es frecuentemente utilizada para determinar la presencia de displasia de cadera tanto en la presentación inicial como en el seguimiento subsecuente. Un fracaso en la disminución del índice acetabular es a menudo utilizado como indicación de una osteotomía periacetabular en el niño con DDC. A pesar de esto, existe preocupación acerca de la exactitud de su medición.

Diversos estudios han mostrado importante variabilidad tanto inter como intraobservador ${ }^{9}$, la que sería mayor en niños con displasia en comparación con niños sanos ${ }^{10}$. Bolton-Maggs y Crabtree demostraron variaciones interobservador en la medición del índice acetabular en una serie de tres radiografías ${ }^{11}$. Así mismo, Kay y colaboradores ${ }^{9}$ demostraron una variación tanto intra como interobservador en la medición del índice acetabular en 24 pacientes con DDC, la cual llegó a ser entre $6^{\circ}$ y $12^{\circ}$. Los autores sugieren que parte de esta diferencia puede ser explicada por la dificultad en identificar los puntos de referencias óseos. Sin embargo, otro estudio, realizado en cirujanos ortopédicos infantiles, mostró menor variación en las mediciones inter e intraobservadores ${ }^{12}$.

Otros métodos de imagen han sido evaluados como alternativa a la radiografía, tales como la RMI y el artroTAC, sin embargo no se han demostrado beneficios en la exactitud de la medición y su costo efectividad en comparación con la medición de índice acetabular en radiografías de screening ${ }^{13}$.

Este estudio busca evaluar la variabilidad existente, tanto intra como interobservador, asociada a la medición del índice acetabular entre profesionales médicos en pacientes sometidos a radiografía de pelvis AP de screening de DDC.

\section{Material y Método}

Fueron obtenidas cien radiografías AP de pelvis, de 100 pacientes, tomadas como screening de DDC en mayo de 2010, todas las cuales fueron realizadas en pacientes de $4 \pm 1$ mes de edad.

Estas radiografías fueron evaluadas por 4 examinadores (un médico general, un médico radiólogo, un cirujano ortopédico infantil y un pediatra), realizándose la medición del índice acetabular en forma manual, en cada una de las caderas. Cada evaluador realizó las mediciones de las 100 radiografías (200 caderas) en 3 períodos de tiempo distintos, con al menos 1 mes de separación. Existió un total de 2.400 mediciones (100 radiografías, ambas caderas, 4 examinadores, en tres tiempos diferentes). Todas las mediciones del índice acetabular fueron realizadas sin instrucciones y a cada examinador se le entrego un lápiz y un goniómetro para su utilización.

Los resultados obtenidos fueron recolectados y analizados. El análisis estadístico fue realizado en el programa SPSS versión 2.0 para Mac. Se realizó un análisis de fiabilidad a través del Alfa de Cronbach, el que indicó que la escala es fiable y el método reproducible. Se utilizó el coeficiente de correlación intraclase (CCI) para evaluar la concordancia de las mediciones de un mismo observador en distintos tiempos y para evaluar la concordancia de los valores obtenidos en las mediciones de distintos observadores (médico general, cirujano ortopédico infantil, radiólogo y pediatra). Los valores de CCI pueden variar desde 0 hasta +1 , el valor 0 indica ausencia de concordancia, mientras que un valor de +1 indica una concordancia lineal perfecta. Se consideró un valor de $\mathrm{CCI}>0,80$ como satisfactorio. 
Tabla 1. Concordancia intra observador en mediciones de índice acetabular

\begin{tabular}{|lcccc|}
\hline Concordancia intra observador & $\mathbf{C C l}$ & IC (95\%) & Alfa de Cronbach & Error alfa \\
\hline Médico general & 0,955 & $0,943-0,965$ & 0,985 & 0,314 \\
Radiólogo & 0,959 & $0,948-0,968$ & 0,987 & 0,288 \\
Cirujano ortopédico infantil & 0,972 & $0,964-0,978$ & 0,991 & 0,288 \\
Pediatra & 0,928 & $0,902-0,949$ & 0,976 & 0,315 \\
\hline
\end{tabular}

Tabla 2. Concordancia inter observador en mediciones de índice acetabular

\begin{tabular}{|lcccc|}
\hline Concordancia inter observador & CCI & IC (95\%) & Alfa de Cronbach & Error alfa \\
\hline 1er tiempo & 0,851 & $0,805-0,890$ & 0,957 & 0,313 \\
2do tiempo & 0,869 & $0,828-0,904$ & 0,963 & 0,295 \\
3er tiempo & 0,900 & $0,867-0,933$ & 0,972 & 0,295 \\
\hline
\end{tabular}

\section{Resultados}

Fueron evaluadas 100 radiografías de pelvis de pacientes de $4 \pm 1$ mes de edad, las que fueron examinadas por 4 médicos de diferentes especialidades (un médico general, un radiólogo, un cirujano ortopédico infantil y un pediatra). En todas estas radiografías fue medido el índice acetabular de cadera derecha e izquierda por 4 médicos en 3 tiempos diferentes, para un total de 2.400 mediciones.

Variación intraobservador: Fueron calculados los rangos entre las tres mediciones realizadas por cada uno de los evaluadores. De las 800 radiografías medidas (4 evaluadores con 200 mediciones cada uno), el promedio de rangos para las tres mediciones de la misma radiografía por el mismo evaluador fue de $1,5^{\circ}$. En sólo 3 caderas se obtuvo un rango intra observador de $6^{\circ}$ y en ningún caso se obtuvo diferencias mayores a $6^{\circ}$.

El coeficiente de correlación intraclase entre las mediciones de IA fue $>0,80$ en todas las mediciones, siendo mayor en el cirujano ortopédico infantil (CCI 0,972) y menor en el pediatra (CCI 0,928) (tabla 1). El análisis de fiabilidad a través del Alfa de Cronbach fue $>0,90$ para todos los observadores, indicando que la escala es fiable (tabla 1).

Variación interobservador: Fueron comparadas las evaluaciones realizadas por los cuatro examinadores en los tres tiempos dife- rentes. La variabilidad interobservador fue de $3,4^{\circ}$ para los diferentes tiempos, sin diferencias entre ellos. En 73 caderas se obtuvo un rango inter observador mayor o igual a $6^{\circ}$, lo que corresponde a un $12 \%$ de las mediciones, y en ningún caso se obtuvo diferencias mayores a $12^{\circ}$.

El coeficiente de correlación intraclase entre las mediciones de IA fue $>0,80$ para los tres tiempos, con una mejor concordancia en la segunda y tercera evaluación, sin llegar a ser diferencias estadísticamente significativas (tabla 2). El análisis de fiabilidad a través del Alfa de Cronbach fue $>0,90$ para los tres tiempos de medición, indicando que la escala es fiable (tabla 2).

\section{Discusión}

Dado la importancia de la detección temprana y la intervención oportuna en pacientes con DDC, se ha prestado mayor atención en casos sutiles de displasia acetabular, por lo que la precisión en la medición de los índices radiográficos es fundamental.

El índice acetabular (ángulo acetabular) continúa jugando un rol esencial en la evaluación radiológica de la cadera displásica y su evolución. La incapacidad de este índice de normalizarse con el tiempo puede ser usado como indicación para cirugía periacetabular en el paciente con displasia. 
A pesar de su uso masivo, existen dudas respecto a su confiabilidad ${ }^{9-12}$. Potenciales fuentes de error en la obtención de la medición del índice acetabular incluyen la elección del borde lateral de la porción osificada del acetábulo, la ubicación adecuada de la línea de Hilgenreiner, las rotaciones en la imagen y el goniómetro utilizado, las que no fueron incluidas en este estudio.

Este estudio demuestra una alta concordancia entre los diferentes profesionales tanto inter como intraobservador en la medición del índice acetabular en radiografías de pelvis AP, tomadas a modo de screening de DDC.

En cuanto al análisis de fiabilidad, la obtención de un Alfa de Cronbach cercano a 1, indica una técnica con resultados consistentes y fiables, lo que la hace reproducible como técnica de screening.

Cuando se evaluaron las tres mediciones de una radiografía por cada uno de los examinadores, las mediciones variaron por 6 grados en sólo 3 mediciones $(0,75 \%)$ sin observarse diferencias mayores a $6^{\circ}$. En las mediciones entre los cuatro médicos, se encontró una diferencia de $6^{\circ}$ o más en 73 de ellas (12\%) sin diferencias de al menos $12^{\circ}$, lo que difiere significativamente con lo reportado en la literatura ${ }^{9}$.

Es importante destacar que todas las radiografías utilizadas en el estudio correspondieron a radiografías de pacientes de $4 \pm 1$ meses lo que disminuye la variabilidad otorgada por la osificación del borde acetabular. Además, las 100 radiografías elegidas fueron obtenidas de modo consecutivo dentro de las radiografías tomadas durante el mes de mayo de 2010. Dentro de estas no existió ninguna cadera luxada, subluxada o con displasia importante, lo que también puede haber contribuido a un mejor resultado en el análisis, ya que como ha sido demostrado, existe una mayor variabilidad en la medición de caderas con displasia respecto a caderas sanas ${ }^{10}$.

Otra variable que puede haber influido en los resultados consiste en que fueron excluidas del estudio radiografías que se encontraran rotadas de forma importante a criterio del cirujano ortopédico infantil, lo que también ha sido reportado como factor de error a la hora de realizar las mediciones del índice acetabu- $\operatorname{lar}^{14}$. En nuestra muestra fueron excluidas 3 radiografías de aquellas revisadas, quedando 100 radiografías incluidas en el estudio.

Las radiografías medidas en este estudio corresponden a radiografías digitales impresas en papel y medidas a mano con goniómetro, por lo que no es posible evaluar errores que pudieran provenir de mediciones electrónicas con distintos programas.

La variabilidad interobservador fue mayor que la intraobservador, lo que es concordante con lo referido en la literatura ${ }^{9-12}$.

Dado que la evolución de las mediciones del índice acetabular es fundamental para la toma de decisiones terapéuticas, varias sugerencias pueden ser inferidas de este trabajo. En primer lugar, para minimizar la variabilidad en la evaluación del índice acetabular, un mismo examinador debiera ser quien mida todas las radiografías de un determinado paciente. Segundo, las radiografías previas deben ser analizadas con el objeto de elegir los mismos puntos de referencia para realizar la medición del índice acetabular. Tercero, pudiera ser posible que la utilización del mismo goniómetro y lápiz disminuyera la variabilidad aún más, lo que no fue evaluado en este trabajo. Estos factores han mostrado minimizar la variabilidad en la evaluación del ángulo de Cobb en las radiografías de pacientes con escoliosis ${ }^{15}$.

Consideramos que es importante destacar la gran concordancia en las diferentes mediciones por los distintos médicos, lo que permite una adecuada identificación de los pacientes que se beneficiarán de un tratamiento oportuno. Esto nos entrega una gran herramienta de trabajo ya que las radiografías corresponden a un examen sencillo, barato, fácil de obtener, al alcance de toda la población, y prácticamente inocuo ya que la dosis de radiación utilizada es ínfima. Este es un examen que tendría la posibilidad de ser interpretado en forma confiable por cualquier médico entrenado o experimentado, lo que entrega ventaja respecto a las ecografías de cadera.

\section{Conclusiones}

Las radiografías de pelvis AP son un buen elemento de screening para DDC en lactantes. 
En radiografías sin rotación, las mediciones obtenidas tienen una alta concordancia tanto inter como intra observador en aquellos médicos que en su práctica profesional realizan estas evaluaciones: médico general, cirujano ortopédico infantil, radiólogo y pediatra.

\section{Referencias}

1.- Raimann A: Enfermedad Luxante de Cadera, Santiago, Editorial Iku 2003 pág 19.

2.- Guía clínica Displasia Luxante de Caderas, Santiago, MINSAL 2010 pag 7.

3.- Holen KJ, Terjesen T, Tegnander A, Bredland T, Saether $O D$, Eik-Nes $S H$ : Ultrasound screening for hip dysplasia in newborns. J Pediatr Orthop 1994; 14: 667-71.

4.- Woolacott NF, Puhan MA, Steurer J, Kleijnen J: Ultrasonography in screening for developmental dysplasia of the hip in newborns: systematic review. BMJ. 2005; 330: 1413-1418.

5.- U.S. Preventive Services Task Force: Screening for developmental Dysplasia of the Hip. 2006.

6.- Arce J, García G: Displasia del desarrollo de caderas ¿radiografía o ultrasonografía? ¿a quiénes y cuándo? Rev Chil Pediat V. 71 n. 4 Santiago Jul 2000.

7.- Tonnis $D$ : Normal values of the hip joint for the evaluation of X-rays in children and adults. Clin Orthop. 1976; 119: 39-47.
8.- Tonnis D: Congenital dysplasia and dislocation of the hip in children and adults (Telger TC, trans.). London: Springer-Verlag, 1987: 100-42.

9.- Kay RM, Watts HG, Dorey FJ: Variability in the assessment of acetabular index. J Pediatr Orthop. 1997; 17: 170-3.

10.- Skaggs DL, Kaminsky C, Tolo VT, Kay RM, Reynolds $R$ : Variability in measurement of acetabular index in normal and dysplastic hips, before and after reduction. J Pediatr Orthop. 1998; 18: 799-801.

11.- Bolton-Maggs BG, Crabtree SD: The opposite hip in congenital dislocation of the hip. J Bone Joint Surg [Br] 1983; 65: 279-84.

12.- Spatz D, Reiger M, Klaumann M, Miller F, Stanton R, Lipton $G$ : Measurement of Acetabular Index Intraobserver and Interobserver Variation. J Pediatr Orthop 1997; 17 (2): 174-5.

13.- Pirpiris M, Payman KR, Otsuka NY: The Assessment of Acetabular Index. Is There Still a Place for Plain Radiography? J Pediatr Orthop. 2006; 26: 310-5.

14.- Van der Bom M, Groote M, Vincken K, Beek F, Bartels L: Pelvic Rotation and Tilt Can Cause Misinterpretation of the Acetabular Index Measured on Radiographs. Clin Orthop Relat Res 2011; 469: 1743-9.

15.- Morrissy RT, Goldsmith GS, Hall EC, Kehl D, Cowie $G H$ : Measurement of the Cobb angle on radiographs of patients who have scoliosis. J Bone Joint Surg (Am) 1990; 72: 320-7. 